\title{
Active destruction of defective ribosomes by a ubiquitin ligase involved in DNA repair
}

\author{
Alan G. Hinnebusch ${ }^{1}$ \\ Laboratory of Gene Regulation and Development, Eunice Kennedy Shriver National Institute of Child Health and Human \\ Development, National Institutes of Health, Bethesda, Maryland 20892, USA
}

Progression of DNA replication forks through damaged DNA requires a ubiquitin ligase comprised of the cullin Rtt101, the RING finger protein Hrt1, and the adaptor protein Mms1. Rtt101 and Mms1 were implicated recently by Fujii and colleagues (pp. 963-974) in the degradation of catalytically inactive mutant $25 \mathrm{~S}$ ribosomal RNAS (rRNAs) in mature 60S ribosomal subunits, a process that requires ubiquitin and is accompanied by ubiquitination of $60 \mathrm{~S}$ components. It now seems likely that the same ubiquitin ligase is enlisted to deal with defective rRNA and damaged DNA.

\section{A panoply of RNA surveillance pathways}

In addition to tightly regulating expression of mRNAs and the amounts and activities of the protein synthetic machinery used to translate mRNAs into proteins, cells must deal with the formation of defective mRNAs with the potential to produce aberrant proteins with harmful effects on cell physiology. The best-studied mRNA surveillance pathway, known as nonsense-mediated decay (NMD), recognizes premature stop codons introduced into the coding sequences of mRNA by mutations, errors in transcription or mRNA splicing, or chemical or radiation damage of RNA, and then targets such mRNAs for degradation during the process of translation. Beginning with studies in the budding yeast Saccharomyces cerevisiae, where NMD was first uncovered, many trans-acting factors responsible for identifying the stop codon, distinguishing it from a normal stop codon, and recruiting the RNA degradation machinery, have been identified and studied intensively. Studies of budding yeast have also identified distinct mRNA decay mechanisms that specifically target truncated mRNAs lacking a stop codon (NSD, nonstop decay) or mRNAs with a strong secondary structure that block ribosome progression (NGD, no-go decay) (Doma and Parker 2007).

[Keywords: Ubiquitin; ribosome; genotoxic stress; quality control; rRNA]

${ }^{1}$ Correspondence.

E-MAIL ahinnebusch@nih.gov; FAX (301) 496-6828.

Article is online at http://www.genesdev.org/cgi/doi/10.1101/gad.1800509.
The structural RNAs that comprise the translational apparatus also undergo several levels of quality control to prevent defective tRNAs or ribosomal RNAs (rRNAs) from accumulating and generating translational errors and competing with their functional counterparts for amino acids, soluble translation factors, and mRNAs. Two pathways have been identified that target improperly modified, processed, or folded tRNAs for degradation. The precursor of initiator tRNA ${ }^{\text {Met }}$ lacking methylation at residue A58, which is inefficiently processed, is targeted for degradation in the nucleus by the exosome, which is a complex of $3^{\prime}$-to-5' exonucleases, following polyadenylation of the defective tRNA by Trf4 of the TRAMP4 (Trf/Air/Mrt4) polyadenylation complex (Kadaba et al. 2004, 2006; LaCava et al. 2005; Vanacova et al. 2005). Several otherwise mature tRNAs lacking different base modifications are degraded in the cytoplasm by the "rapid tRNA decay" (RTD) pathway through the combined action of the $5^{\prime}$-to-3' exonucleases Rat1 and Xrn1 (Chernyakov et al. 2008). Quality control is also exerted at the step of tRNA export from the nucleus, which is denied to tRNAs that cannot be aminoacylated by nucleus-associated aminoacyl-tRNA synthetases (Lund and Dahlberg 1998; Sarkar et al. 1999; Grosshans et al. 2000; Hopper and Shaheen 2008).

The biogenesis of ribosomes involves a complex pathway in which the $35 \mathrm{~S}$ pre-rRNA is processed to mature $18 \mathrm{~S}, 5.8 \mathrm{~S}$, and 25S rRNAs, which are assembled with the large number of ribosomal proteins constituting the small (40S) or large (60S) ribosomal subunits. Most of these steps occur in the nucleolus, but the final ones take place in the nucleoplasm or after export of nascent subunits to the cytoplasm. The complexity of the processing and export pathway and the large number of factors involved provide the potential for errors and generation of defective preribosomal subunits. In mutants impaired for a ribosome processing factor, the export of immature or defective subunits can be blocked (Tschochner and Hurt 2003). The pre-rRNAs in defective ribosomal subunits that cannot be exported can be degraded by the nuclear exosome (Allmang et al. 2000; Dez et al. 2006). In one such mutant (sda1-2) where the export of large and 
small subunits is impaired, degradation of the $35 \mathrm{~S}$ and $27 \mathrm{~S}$ pre-rRNAs and mature 25S rRNA by the exosome is stimulated by the same TRAMP4 polyadenylation complex involved in tRNA quality control, and this surveillance mechanism appears to be localized to a nucleolar structure termed the No-body. It was speculated that preribosomes constitutively recruit TRAMP complex as a "fail-safe" mechanism that enables degradation of rRNA if TRAMP is not displaced in later steps of ribosome maturation. This would ensure that defective preribosomes do not accumulate and sequester limiting processing factors needed to ensure rapid ribosome biogenesis under conditions optimal for cell growth (Dez et al. 2006).

\section{Quality control for mature ribosomes}

Interestingly, there is also evidence for a surveillance mechanism that targets functionally impaired, mature ribosomes already resident in the cytoplasm. LaRiviere et al. (2006) reported that mutations producing nucleotide substitutions in $18 \mathrm{~S}$ or $25 \mathrm{~S}$ rRNAs that debilitate the decoding or peptidyl transferase center (PTC), respectively, confer more rapid turnover in the cytoplasm with little effect on the biogenesis of the defective subunits or the stability of wild-type subunits in the same cells. It was proposed that an active process, dubbed nonfunctional rRNA decay (NRD), acts to eliminate the rRNA component of such defective, mature ribosomes. It was further suggested that NRD would allow cells to rid themselves of ribosomal subunits in which the rRNA was damaged by alkylating agents, oxidative damage, or UV irradiation, which might be cumulative with age (LaRiviere et al. 2006). However, the molecules that participate in NRD were unknown, and it has been speculated that defective ribosomes not actively involved in elongation might be eliminated by the passive mechanism of increased susceptibility to nucleases (Doma and Parker 2007).

The study by Fujii et al. (2009) in this issue of Genes \& Development demonstrates that an active mechanism exists to eliminate nonfunctional ribosomes and identifies two proteins previously unconnected to ribosomes or translation that are required specifically for degradation of $25 \mathrm{~S}$ rRNA in defective $60 \mathrm{~S}$ subunits. These factors, Mms1 and Rtt101, are components of an E3 ubiquitin ligase complex described previously for its role in DNA repair. Fujii et al. (2009) detected ubiquitin-conjugated proteins in ribosomal fractions containing nonfunctional 25S rRNAs, presumed to be ribosomal components modified by the NRD, whose abundance is dependent on Mms1 and Rtt101. They further provided evidence that ubiquitin-dependent protein degradation is crucial for 25S rRNA NRD.

As in the discovery of other RNA quality control mechanisms mentioned above, the power of yeast genetics made these new developments possible. The discovery and analysis of NRD has relied on prior construction of specialized yeast strains (Nogi et al. 1991; Venema et al. 1995) in which expression of wild-type rRNA can be repressed at elevated temperature $\left(37^{\circ} \mathrm{C}\right)$ by a temperaturesensitive mutation in a subunit of RNA Polymerase I (responsible for rDNA transcription) and harboring an rDNA allele under the control of the glucose-repressible, galactose-inducible $G A L 7$ promoter for RNA Polymerase II (responsible for mRNA synthesis). Viability of this strain at $37^{\circ} \mathrm{C}$, where the endogenous rDNA cannot be transcribed, depends on rRNA expressed from the $P_{G A L 7^{-}}$ $R D N$ allele. Accordingly, the strain grows on galactose at $37^{\circ} \mathrm{C}$, where $P_{G A L 7}-R D N$ is expressed, but dies on glucose at $37^{\circ} \mathrm{C}$, where $P_{G A L 7}-R D N$ is repressed. The functionality of substituted rRNAs expressed from mutant $P_{G A L 7^{-}}$ $R D N$ alleles is assessed by scoring growth on galactose at $37^{\circ} \mathrm{C}$. An important embellishment of the approach involved introduction of an innocuous 18-nucleotide (nt) tag into the 25S rRNA coding sequences to allow its product to be distinguished from endogenous rRNA by Northern blot or RT-PCR analyses (Musters et al. 1989).

Using such an engineered yeast strain, Fujii et al. (2009) studied substitutions in the PTC, A2451U and C2452G, that render 25S rRNA nonfunctional in the growth assay. The nonfunctionality of C2457G was established previously (Rakauskaite and Dinman 2006) and LaRiviere et al. (2006) showed that a different substitution of A2451 (to G) was nonfunctional and conferred NRD, whereas C2452U was viable and did not destabilize 25S rRNA. Fujii et al. (2009) wished to address whether the destabilization conferred by A2451G in the previous study was unique to that alteration or whether any functional impairment of the PTC would trigger NRD. Indeed, both A2451U and C2452G lowered the steady-state amount of mature tagged $25 \mathrm{~S}$ rRNA by decreasing its half-life. The mutant 25S rRNAs were incorporated into 60S subunits, which were detected in the cytoplasm-consistent with completion of 60S biogenesis-but displayed impaired incorporation into polysomes, the translationally active pool of $80 \mathrm{~S}$ ribosomes. Thus, the stability of mature $25 \mathrm{~S}$ rRNA in defective 60S subunits, rather than synthesis or processing of pre-rRNA, was being reduced-the hallmark of NRD.

\section{Nonfunctional 60S decay requires ubiquitin ligase subunit Mms1}

Armed with these reagents, Fujii et al. (2009) used another tool in the yeast genetics armamentarium, the library of viable haploid deletion mutants (Winzeler et al. 1999), to identify trans-acting factors required for NRD. They transformed all $\sim 5000$ deletion strains with the $P_{G A L 7}-R D N$ allele expressing the A2451U 25S rRNA and screened colonies by hybridization for elevated amounts of this normally unstable mutant rRNA. They began by excluding a requirement for key factors involved in other RNA surveillance systems, NMD, NGD, NSD, RTD, and TRAMP/exosome, as well as a recently discovered ribosome degradation pathway operating under starvation conditions, dubbed ribophagy (Kraft et al. 2008). By random screening, they identified the mms1s strain as being defective in rapid degradation of mature mutant 
25S rRNAs harboring PTC substitutions without altering levels of the cognate 35S pre-RNAs. Moreover, mms1A appeared to increase the proportion of the corresponding mutant 60S subunits associated with $40 \mathrm{~S}$ subunits in $80 \mathrm{~S}$ ribosomes and, for the A2451U mutant, even in polysomes. These findings indicate that Mms1 is required to prevent the accumulation of defective $60 \mathrm{~S}$ subunits in the cytoplasm able to compete with functional $60 \mathrm{~S}$ for joining with $40 \mathrm{~S}$ subunits. It is intriguing that another NRD substrate with the A1492C substitution in the decoding site of $18 \mathrm{~S}$ rRNA (LaRiviere et al. 2006) was not stabilized by mms $1 \Delta$, implying that NRD for $40 \mathrm{~S}$ and 60S subunits employs distinct factors.

Prior to this work, it was formally possible to argue that mutant $25 \mathrm{~S}$ rRNAs that undergo rapid degradation are unable to provide 25S rRNA function in living cells, because they are unstable instead of functionally impaired. If this argument was taken to the extreme, one could even question the existence of NRD as an active process. This possibility now seems very remote for two reasons. First, Fujii et al. (2009) were able to prove (at least for the A2451U and C2452G mutants) that these 25S rRNAs are inadequate for cell growth even when allowed to accumulate to near wild-type levels in the mms1s background. Thus, these 25S rRNAs are nonfunctional and not merely unstable. Second, they identified a nonribosomal factor-i.e., Mms1-required for the active process of degrading defective 25S rRNAs in mature 60S subunits. Moreover, Fujii et al. (2009) could show that expressing the A2451U and U2585A rRNAs is harmful to cell growth in mms1A cells that cannot rid themselves of mutant 60S subunits. As LaRiviere et al. (2006) pointed out, it is interesting that Escherichia coli seems to lack a comparable surveillance system and, hence, is poisoned by expressing comparable defective rRNAs (Green et al. 1997).

\section{A ubiquitin ligase core governs multiple responses to nucleic acid damage}

Mms1 has a much longer history in yeast genetics, being identified originally as a factor required for protection against DNA damage during replication and normal resistance to the alkylating agent methyl methanesulfonate (MMS) (Prakash and Prakash 1977; Hryciw et al. 2002). On the basis of genetic and protein-protein interaction data, it was assigned recently to a group of factors called the Mms22 module, including Mms22, Rtt101 (also known as Cul8), and Rtt107, which function together to protect against, or repair, DNA damage (Pan et al. 2006). Rtt101 is a cullin protein that interacts in vivo with the RING finger-containing protein Hrtl (also known as Roc1) to comprise a ubiquitin ligase (E3) complex that is capable of activating polyubiquitin chain formation by the E2 enzyme Cdc34 in vitro (Michel et al. 2003). Rtt101 also binds to Mms1 (Suter et al. 2007), which bridges an interaction with Mms22 under conditions of DNA damage (MMS) in vivo (Zaidi et al. 2008). Several lines of evidence suggest that the Rtt101-Mms1Mms22 ubiquitin ligase complex acts to permit the com- pletion of DNA replication after replication fork arrest induced by DNA damage (Luke et al. 2006; Zaidi et al. 2008).

Fujii et al. (2009) examined the other members of the Mms22 module and found that deleting RTT101 also conferred defects in NRD comparable with those seen for $m m s 1 \Delta$; i.e., strongly stabilizing the A2451U and C2452G mutants, partially stabilizing the U2585A mutant, and having no effect on the A1492C 18S rRNA mutant. Given the established function of Rtt101 as a ubiquitin ligase, they went on to examine whether Mms1 and Rtt101 function in NRD by mediating ubiquitination of components of defective 605 subunits. Supporting this idea, ribosomes purified from cells expressing the defective $25 \mathrm{~S}$ rRNAs were found to be enriched in ubiquitinated proteins in a manner dependent on both Mms1 and Rtt101. And ubiquitinated ribosomes purified from the total pool of ribosomes were enriched in tagged 25S rRNA when it contained the debilitating mutations compared with wild-type tagged 25S rRNA. Finally, to provide evidence that NRD is a ubiquitinmediated process, Fujii et al. (2009) demonstrated that overexpressing a nonfunctional $\mathrm{N}$-terminal myc-tagged form of ubiquitin, known to impair ubiquitin-dependent proteolysis, stabilized the defective A2451U 25S rRNA. Thus, it is very likely that Mms1 and Rtt101 function in NRD as components of an E3 complex that ubiquitinates components (yet to be identified) of defective mature 60S subunits in a manner that evokes degradation of the mutant 25S rRNA.

Because NRD does not require Mms22, Fujii et al. (2009) propose that Rtt101, Mms1, and Cdc34 comprise a core ubiquitin ligase complex that uses different adaptor proteins to execute distinct functions. (It seems likely to me that Hrt1 also belongs to this core complex.) Mms22 would be recruited by the core E3 components during DNA damage to mediate ubiquitination of an unknown substrate involved in DNA repair (Fig. 1A), whereas other unknown adaptor proteins would enable ubiquitination of $60 \mathrm{~S}$ ribosomal components in the NRD (Fig. 1C). Interestingly, Rtt101 and Mms1 also interact with Crt10, a transcriptional regulator of ribonucleotide reductase (RNR) induced by DNA damage, and Zaidi et al. (2008) have speculated that Crt10 could also stimulate RNR activity by targeting an RNR inhibitor for ubiquitination and degradation, as occurs in fission yeast (Fig. 1B; Zaidi et al. 2008). Fujii et al. (2009) suggest that it makes sense to enlist a common core ubiquitin ligase complex, which they dub GUARD (genotoxic stress-related ubiquitin ligase associated with RNA and DNA damage) to mediate responses to both DNA damage and the NRD, as genotoxic agents can be expected to damage rRNA as well as DNA. Given that Mms1 and Rtt101 are dispensable for rapid degradation of the A1492C mutant $18 \mathrm{~S}$ rRNA, another ubiquitin ligase or a nonubiquitin-based regulatory mechanism must handle degradation of defective $40 \mathrm{~S}$ subunits. In this regard, it would be useful to know whether rapid degradation of the mutant $18 \mathrm{~S}$ rRNA is blocked by overexpressing the defective form of ubiquitin. 

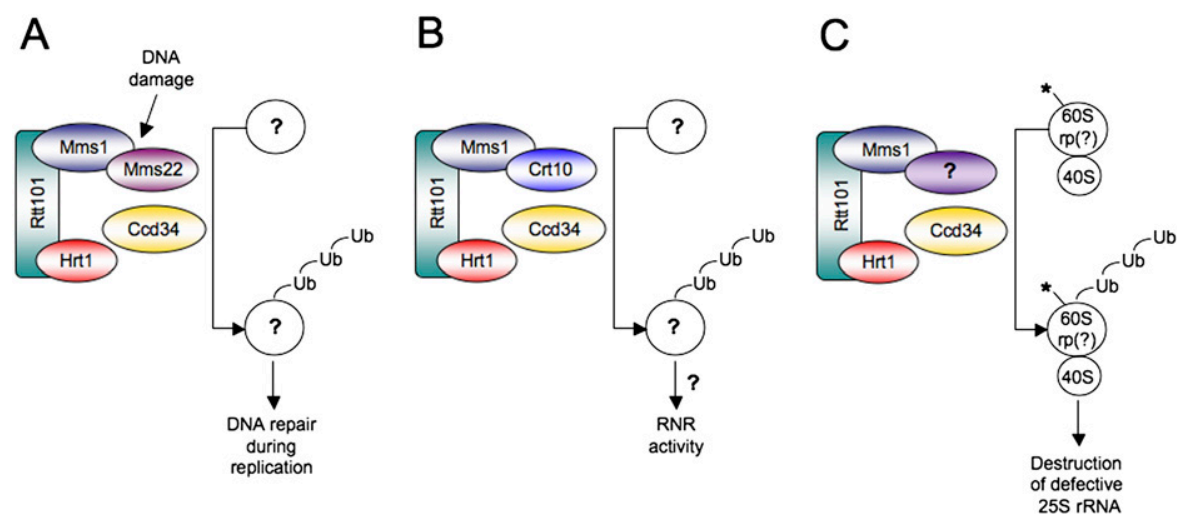

Figure 1. Model describing the use of a common ubiquitin ligase complex for multiple responses to conditions that damage nucleic acids. The cullin Rtt101, RING finger-containing protein Hrt1, and adaptor protein Mms1 form a core ubiquitin ligase complex (E3) that functions with ubiquitin-conjugating enzyme (E2) Cdc34. (A) The core E3 recruits Mms22 in response to DNA damage and ubiquitinates an unknown protein in a manner that stimulates DNA repair during replication. $(B)$ The core E3 recruits Crt10 to ubiquitinate an unknown factor in a manner that eliminates a hypothetical inhibitor of RNR activity (Zaidi et al. 2008). (C) The core E3 recruits an unknown adaptor that promotes ubiquitination of one or more unknown 60S subunit ribosomal proteins (rp) in functionally defective 60S subunits (marked by an asterisk), leading to the destruction of the 25S rRNA. Modified by permission from Macmillan Publishers Ltd. (Zaidi et al. [2008] @2008) as suggested by Fujii et al. (2009).

\section{How are defective 60S subunits detected and degraded?}

A key unanswered question is how the defective 60S subunits are recognized in the cell. It is difficult to imagine that catalytically defective 60S subunits would be recognized in the form of free subunits, because $60 \mathrm{~S}$ subunits have no known function until they join with the preinitiation complex assembled on the small (40S) subunit at the start codon to form the 80S initiation complex that is ready for peptide bond formation. In mms14 cells, where the defective 60S subunits accumulate, those containing the A2451U mutant rRNA were present in polysomes, albeit reduced in abundance and average size compared with polysomes containing only wild-type $60 \mathrm{~S}$ subunits. If the mutant $80 \mathrm{~S}$ ribosomes are capable of elongating but do so more slowly than wild-type ribosomes, one expects to observe larger, not smaller, polysomes than occur in wild-type cells, as the defective ribosomes should impede the progression of wild-type ribosomes translating the same mRNA. If the mutant $80 \mathrm{~S}$ ribosomes cannot make peptide bonds and remain stuck at the start codon, then all of the defective $60 \mathrm{~S}$ subunits should accumulate as $80 \mathrm{~S}$ monosomes, because the wild-type elongating ribosomes already present on the mRNA would finish translation and dissociate from the mRNA. On the other hand, if the mutant 80 S ribosomes cannot elongate but dissociate relatively quickly from the initiation region, then they will simply decrease the rate of initiation by wild-type ribosomes and yield the combination of smaller polysomes and increased monosomes observed by Fujii et al. (2009). Hence, perhaps the elongation-defective $80 \mathrm{~S}$ ribosomes are identifiable because they spend a moderately prolonged amount of time bound to mRNA at the start codon with methionyl-tRNA in the P-site and EF-1A-aminoacyl-tRNA-GTP ternary complex in the A-site.
In wild-type cells, Fujii et al. (2009) found that a much greater proportion of the A2451U subunits accumulated as free 60S subunits, which could be explained by proposing that the ubiquitination that occurs in the context of $80 \mathrm{~S}$ monosomes diminishes the ability of the modified 60S subunits to participate in subsequent rounds of subunit joining with the wild-type 40S subunits. Based on their images of GFP-tagged 60S subunits in wild-type cells expressing defective 25S rRNA, Fujii et al. (2009) speculate that the modified 60S subunits are sequestered in bodies associated with the nucleus in order to be disassembled. It is important to ask whether mutations or inhibitors that bar proteasome function will disrupt NRD to address whether proteasomal degradation of ribosomal proteins is being triggered by ubiquitination and is necessary to elicit destruction of the rRNA, and also to identify the nuclease(s) involved in the latter.

Finally, it is intriguing that mature ribosomes are degraded by autophagy during nitrogen starvation in a process termed ribophagy, wherein destruction of the large subunit specifically is enhanced by ubiquitin protease Ubp3 and its cofactor Bre5. As ubiquitinated proteins were more adundant in ribosomes purified from ubp3s versus wild-type cells, it appeared likely that deubiquitination of ribosomal components was directly involved in ribophagy. It might seem counterintuitive that deubiquitination is required for $60 \mathrm{~S}$ destruction by ribophagy, whereas ubiquitination triggers breakdown of defective subunits in the NRD. However, it is possible that ubiquitination also targets the 60S for degradation by ribophagy and that deubiquitination by Ubp $3 /$ Bre 5 is needed to complete the process (Kraft et al. 2008). The ubiquitin ligase(s) essential for ribophagy have not been identified. It might be worthwhile to determine whether the GUARD complex is involved. It is also of interest to consider whether any deubiquitinating activities other than Ubp3/Bre5 are needed for the NRD. 


\section{Acknowledgments}

This work was supported in part by the Intramural Research Program of the NIH.

\section{References}

Allmang, C., Mitchell, P., Petfalski, E., and Tollervey, D. 2000. Degradation of ribosomal RNA precursors by the exosome. Nucleic Acids Res. 28: 1684-1691.

Chernyakov, I., Whipple, J.M., Kotelawala, L., Grayhack, E.J., and Phizicky, E.M. 2008. Degradation of several hypomodified mature tRNA species in Saccharomyces cerevisiae is mediated by Met22 and the $5^{\prime}-3^{\prime}$ exonucleases Rat1 and Xrn1. Genes \& Dev. 22: 1369-1380.

Dez, C., Houseley, J., and Tollervey, D. 2006. Surveillance of nuclear-restricted pre-ribosomes within a subnucleolar region of Saccharomyces cerevisiae. EMBO J. 25: 1534-1546.

Doma, M.K. and Parker, R. 2007. RNA quality control in eukaryotes. Cell 131: 660-668.

Fujii, K., Kitabatake, M., Sakata, T., Miyata, A., and Ohno, M. 2009. A role for ubiquitin in the clearance of nonfunctional rRNAs. Genes \& Dev. (this issue). doi: 10.1101/gad.1775609.

Green, R., Samaha, R.R., and Noller, H.F. 1997. Mutations at nucleotides G2251 and U2585 of $23 \mathrm{~S}$ rRNA perturb the peptidyl transferase center of the ribosome. J. Mol. Biol. 266: 40-50.

Grosshans, H., Hurt, E., and Simos, G. 2000. An aminoacylationdependent nuclear tRNA export pathway in yeast. Genes \& Dev. 14: 830-840.

Hopper, A.K. and Shaheen, H.H. 2008. A decade of surprises for tRNA nuclear-cytoplasmic dynamics. Trends Cell Biol. 18: 98-104.

Hryciw, T., Tang, M., Fontanie, T., and Xiao, W. 2002. MMS1 protects against replication-dependent DNA damage in Saccharomyces cerevisiae. Mol. Genet. Genomics 266: 848857.

Kadaba, S., Krueger, A., Trice, T., Krecic, A.M., Hinnebusch, A.G., and Anderson, J. 2004. Nuclear surveillance and degradation of hypomodified initiator tRNAMet in S. cerevisiae. Genes \& Dev. 18: 1227-1240.

Kadaba, S., Wang, X., and Anderson, J.T. 2006. Nuclear RNA surveillance in Saccharomyces cerevisiae: Trf4p-dependent polyadenylation of nascent hypomethylated tRNA and an aberrant form of 5S rRNA. RNA 12: 508-521.

Kraft, C., Deplazes, A., Sohrmann, M., and Peter, M. 2008. Mature ribosomes are selectively degraded upon starvation by an autophagy pathway requiring the Ubp3p/Bre5p ubiquitin protease. Nat. Cell Biol. 10: 602-610.

LaCava, J., Houseley, J., Saveanu, C., Petfalski, E., Thompson, E., Jacquier, A., and Tollervey, D. 2005. RNA degradation by the exosome is promoted by a nuclear polyadenylation complex. Cell 121: 713-724.

LaRiviere, F.J., Cole, S.E., Ferullo, D.J., and Moore, M.J. 2006. A late-acting quality control process for mature eukaryotic rRNAs. Mol. Cell 24: 619-626.

Luke, B., Versini, G., Jaquenoud, M., Zaidi, I.W., Kurz, T., Pintard, L., Pasero, P., and Peter, M. 2006. The cullin Rtt101p promotes replication fork progression through damaged DNA and natural pause sites. Curr. Biol. 16: 786-792.

Lund, E. and Dahlberg, J.E. 1998. Proofreading and aminoacylation of tRNAs before export from the nucleus. Science 282: 2082-2085.

Michel, J.J., McCarville, J.F., and Xiong, Y. 2003. A role for Saccharomyces cerevisiae Cul8 ubiquitin ligase in proper anaphase progression. J. Biol. Chem. 278: 22828-22837.
Musters, W., Venema, J., van der Linden, G., van Heerikhuizen, H., Klootwijk, J., and Planta, R.J. 1989. A system for the analysis of yeast ribosomal DNA mutations. Mol. Cell. Biol. 9: 551-559.

Nogi, Y., Yano, R., and Nomura, M. 1991. Synthesis of large rRNAs by RNA polymerase II in mutants of Saccharomyces cerevisiae defective in RNA polymerase I. Proc. Natl. Acad. Sci. 88: 3962-3966.

Pan, X., Ye, P., Yuan, D.S., Wang, X., Bader, J.S., and Boeke, J.D. 2006. A DNA integrity network in the yeast Saccharomyces cerevisiae. Cell 124: 1069-1081.

Prakash, L. and Prakash, S. 1977. Isolation and characterization of MMS-sensitive mutants of Saccharomyces cerevisiae. Genetics 86: 33-55.

Rakauskaite, R. and Dinman, J.D. 2006. An arc of unpaired "hinge bases" facilitates information exchange among functional centers of the ribosome. Mol. Cell. Biol. 26: 89929002.

Sarkar, S., Azad, A.K., and Hopper, A.K. 1999. Nuclear tRNA aminoacylation and its role in nuclear export of endogenous tRNAs in Saccharomyces cerevisiae. Proc. Nat1. Acad. Sci. 96: $14366-14371$.

Suter, B., Fetchko, M.J., Imhof, R., Graham, C.I., Stoffel-Studer, I., Zbinden, C., Raghavan, M., Lopez, L., Beneti, L., Hort, J., et al. 2007. Examining protein-protein interactions using endogenously tagged yeast arrays: The cross-and-capture system. Genome Res. 17: 1774-1782.

Tschochner, H. and Hurt, E. 2003. Pre-ribosomes on the road from the nucleolus to the cytoplasm. Trends Cell Biol. 13: 255-263.

Vanacova, S., Wolf, J., Martin, G., Blank, D., Dettwiler, S., Friedlein, A., Langen, H., Keith, G., and Keller, W. 2005. A new yeast poly(A) polymerase complex involved in RNA quality control. PLOS Biol. 3: e189. doi: 10.1371/journal. pbio.0030189.

Venema, J., Dirks-Mulder, A., Faber, A.W., and Raue, H.A. 1995. Development and application of an in vivo system to study yeast ribosomal RNA biogenesis and function. Yeast 11: 145156.

Winzeler, E.A., Shoemaker, D.D., Astromoff, A., Liang, H., Anderson, K., Andre, B., Bangham, R., Benito, R., Boeke, J.D., Bussey, H., et al. 1999. Functional characterization of the $S$. cerevisiae genome by gene deletion and parallel analysis. Science 285: 901-906.

Zaidi, I.W., Rabut, G., Poveda, A., Scheel, H., Malmstrom, J., Ulrich, H., Hofmann, K., Pasero, P., Peter, M., and Luke, B. 2008. Rtt101 and Mms1 in budding yeast form a CUL4(DDB1)-like ubiquitin ligase that promotes replication through damaged DNA. EMBO Rep. 9: 1034-1040. 


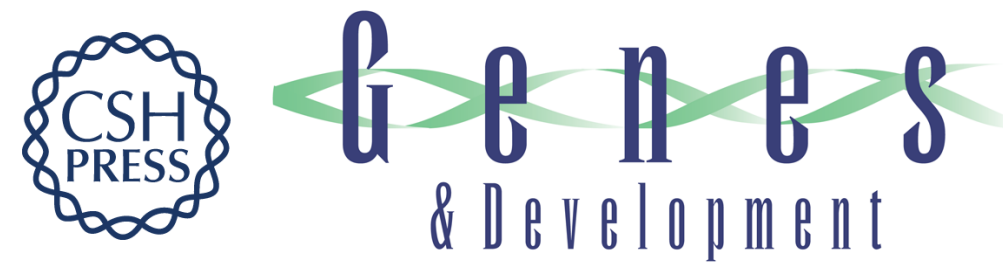

\title{
Active destruction of defective ribosomes by a ubiquitin ligase involved in DNA repair
}

\author{
Alan G. Hinnebusch
}

Genes Dev. 2009, 23:

Access the most recent version at doi:10.1101/gad.1800509

$\begin{array}{ll}\text { References } & \text { This article cites } 29 \text { articles, } 14 \text { of which can be accessed free at: } \\ \text { http://genesdev.cshlp.org/content/23/8/891.full.html\#ref-list-1 }\end{array}$

License

Email Alerting Receive free email alerts when new articles cite this article - sign up in the box at the top Service right corner of the article or click here.

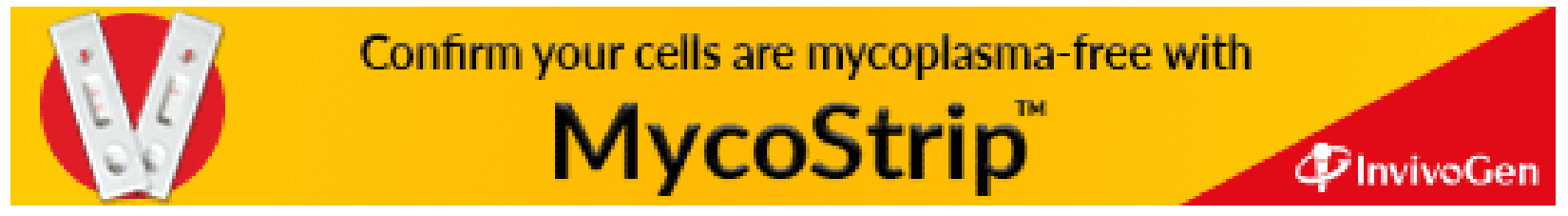

